\title{
EDUCAÇÃO NUTRICIONAL PARA IDOSOS INSTITUCIONALIZADOS NO RECANTO NOSSA SENHORA DO ROSÁRIO EM LIMEIRA, SP
}

\author{
Ariadne Cecílio ${ }^{1}$ \\ Julicristie Machado de Oliveira²
}

resumo

1 Nutricionista na Faculdade de Ciências Aplicadas da Unicamp. E-mail: ariadne.cecilio@hotmail.com

2 Nutricionista. Doutora em Nutrição em Saúde Pública. Professora Doutora na Faculdade de Ciências Aplicadas da Unicamp. E-mail: julicristie.oliveira@fca.unicamp.br 
um para considerações finais e confraternização. Nas duas primeiras semanas, observou-se o cardápio habitual do recanto desde o preparo até o momento em que a refeição foi servida. O valor energético total girou em torno de 1500 kcal a 1600 kcalcom distribuição adequada para macronutrientes, limítrofe ou inferior à recomendada para micronutrientes e fibras, e aumentada para gordura saturada. Somente 10 idosos estavam aptos e se dispuseram a participar da antropometria, sendo cinco classificados com sobrepeso. A atividade com a pirâmide alimentar envolveu oito idosos. O tema hidratação e ingestão de líquidos era o mais aguardado pelo grupo devido à dificuldade em relação a ele. Dezoito idosos se interessaram em participar da atividade. A educação nutricional mostrou-se válida, sendo possível perceber mudanças sutis, embora importantes, logo nas semanas seguintes às atividades aplicadas.

palavras-chave

Educação Alimentar e Nutricional. Nutrição do Idoso. Saúde do Idoso. Saúde do Idoso Institucionalizado.

Segundo dados da Organização Mundial de Saúde (OMS), nos países em desenvolvimento, são considerados idosos todos os indivíduos com idade superior a 60 anos (WHO, 2010). Estimativas da OMS (WHO, 2005) apontam que, até o ano de 2025, o Brasil será o sexto colocado mundial em relação ao número de pessoas idosas (BRASIL, 2012). Embora o envelhecimento populacional brasileiro pudesse ser dado como certo no começo do século XX, devido à queda da mortalidade, somente em meados de 1950 este fenômeno, conhecido como transição demográfica, estabeleceu-se realmente, associando-se ao grande declínio da fecundidade (LEBRÃO, 2007; PAULO et al., 2012).

Em paralelo à transição demográfica, ocorre a transição epidemiológica caracterizada pela diminuição da mortalidade por doenças infecciosas e parasitárias e pelo aumento das doenças crônicas não-transmissíveis (DCNT) (COSTA; THULER, 2012; LEBRÃO, 2007). Os fatores de risco das DCNT são os mesmo em qualquer faixa etária, contudo, em virtude do tempo e hábitos de vida, encontram-se expostos por um período muito maior, por isso é comum que o idoso apresente fatores simultâneos (VITORELI; PESSINI; SILVA, 2005). 
Estudos na área da saúde apontam que a dieta, características ambientais, hábitos de vida e fatores de risco como tabagismo, álcool, obesidade, colesterol, sedentarismo, dentre outros, têm consequências sobre aspectos metabólicos e fisiológicos do organismo de indivíduos idosos. Sendo assim, é possível alcançar, por meio da promoção da saúde e prevenção de doenças, um envelhecimento saudável, preservando a capacidade funcional do idoso pelo maior tempo possível (ALENCAR; BARROS JÚNIOR; CARVALHO, 2008).

Do ponto de vista histórico, o cuidado com o idoso dependente ou que apresentasse algum tipo de incapacidade, seja mental ou funcional, era feito em domicílio por seus familiares. Tal prática, porém, foi se tornando cada vez mais difícil devido a mudanças na rotina familiar e do ingresso da mulher no mercado de trabalho, pois esta, segundo Watanabe (2009), é, na maioria das vezes, a escolhida como cuidadora familiar. Deste modo, a responsabilidade pela assistência passa a ser dividida com o Estado a partir da criação das Instituições de Longa Permanência para Idosos (ILPI). Com isto, percebe-se um crescimento no número de idoso que passa a viver nas ILPI, que, de modo geral, são locais onde esta população tem supridas suas necessidades básicas em relação à moradia, higiene e alimentação (SILVA, 2007; WATANABE, 2009), bem como uma lugar de socialização e criação de vínculos.

O local escolhido para o desenvolvimento desta intervenção educativa é o Recanto dos Idosos Nossa Senhora do Rosário (RINSER), que pertence à Paróquia São Benedito da Diocese de Limeira. Esta paróquia é quarta mais antiga da cidade e foi criada em 1960. O recanto foi fundado em 1995, assiste idosos do sexo masculino e feminino, e conta com um quadro permanente de funcionários composto por administrador, ajudantes gerais, cozinheiras, auxiliares e técnicas em enfermagem e em nutrição, e por enfermeira, além de geriatra e nutricionista voluntários. A manutenção financeira da entidade é realizada por meio da contribuição das famílias dos idosos, de doações e pela realização de eventos para arrecadação de fundos (jantares beneficentes, bazares) (COLÉGIO SÃO BENEDITO, 2014).

Segundo Freire Júnior e Tavares (2005), esta população institucionalizada pode ser excluída socialmente já que se encontra afastada de casa, amigos e familiares. Para Ferreira et al. (2012), viver em uma instituição é poder restabelecer a vida do idoso em sua integralidade uma vez que, nela, o idoso pode criar novos vínculos, principalmente com os demais internos estabelecendo novas relações de amizade, carinho e afeto que alguns não teriam se não estivessem institucionalizados devido ao abandono da família. Sendo assim, quando necessária a institucionalização do idoso, o ideal é que família e instituição trabalhem juntas garantindo que todos seus direitos sejam respeitados. 
Claro que devemos apontar que o inverso também pode ocorrer e o idoso pode se sentir desrespeitado ao ser obrigado a cortar antigos vínculos sociais, o que, segundo Carneiro et al. (2007), pode levar à diminuição da qualidade de vida, da capacidade funcional e da longevidade, associando-se, em alguns casos, a quadros de depressão.

Segundo Ferreira et al. (2012) e Assis (2002), a promoção do envelhecimento saudável se mostra complexa, pois gira em torno não apenas da qualidade de vida, mas também do acesso a serviços de saúde que auxiliem o idoso neste processo. Sendo assim, a educação nutricional é uma das principais ferramentas no que diz respeito à promoção da saúde, modificando hábitos alimentares e dando autonomia para que os indivíduos possam fazer escolhas saudáveis (CRISCUOLO, 2009).

As práticas educativas devem ter como base o diálogo, e, em se tratando da saúde dos idosos, esta abordagem deve ser feita com maior apreço e cuidado para que se crie um vínculo educador-educando, promovendo a autoestima do idoso e devolvendo-lhe o sentido de humanidade (ASSIS, 2002). Considerando o potencial da alimentação em promover melhores condições de saúde (HARDT, et al., 2006), elegeu-se a educação nutricional como estratégia a fim de contribuir com a qualidade de vida dos idosos. Assim, o objetivo deste trabalho foi promover, por meio de atividades de Educação Nutricional, hábitos alimentares saudáveis em um grupo de idosos institucionalizados no RINSER da cidade de Limeira, São Paulo.

\section{Materiais e métodos}

Tratou-se de um estudo de intervenção, do tipo antes e depois, composto por uma amostra de 23 idosos, de ambos os sexos, residentes no RINSER da cidade de Limeira, São Paulo. Inicialmente, foi realizado um diagnóstico e, posteriormente as atividades educativas foram implementadas. Para as atividades de diagnóstico e educação, foram estabelecidas visitas semanais ao longo de sete semanas, totalizando sete encontros, sendo dois para educação nutricional com propostas de atividades semanais realizadas em aproximadamente 60 minutos cada. Primeiramente, foram feitas análises quantitativas e qualitativas das refeições do cardápio habitual no asilo. Para tanto, observou-se desde o pré-preparo até o momento da refeição em si e anotadas, em medidas caseiras, a quantidade ofertada aos idosos. Em um dia da semana, observou-se o lanche da tarde, o jantar e a ceia, e, em um dia da semana seguinte, o desjejum, lanche da manhã e almoço, e todos os idosos participaram desta etapa. Após coleta 
dos dados, analisou-se o cardápio oferecido em relação a macro e micronutrientes com o auxílio do software Avanutri 4.0. Posteriormente, calculou-se a estimativa do valor energético de das refeições que compõem um dia com base no rendimento das preparações e do valor per capta de cada uma delas.

Na terceira semana, realizou-se a aferição do peso corporal e estatura em dez idosos que estavam aptos e que se dispuseram a participar, sendo quatro do sexo masculino e seis do feminino. Utilizou-se balança de referência mecânica com régua antropométrica acoplada da marca Welmy e, para a circunferência abdominal $(\mathrm{cm})$, fita métrica inelástica com precisão de $0,1 \mathrm{~mm}$. Após coletados os dados, calculou-se o Índice de Massa Corporal (IMC). Foram utilizados os pontos de corte do Ministério da Saúde (BRASIL, 2012) para classificar o estado nutricional dos idosos, sendo menor ou igual a $22,0 \mathrm{~kg} / \mathrm{m}^{2}$, baixo peso; maior que 22,0 e menor que $27,0 \mathrm{~kg} / \mathrm{m}^{2}$ eutrófico, e maior ou igual a $27,0 \mathrm{~kg} / \mathrm{m}^{2}$, sobrepeso. Identificou-se, também, o risco de complicações metabólicas associadas com a obesidade, segundo perímetro abdominal, com base nos pontos de corte de circunferência abdominal propostos pela Associação Brasileira para o Estudo da Obesidade e da Síndrome Metabólica (Abeso) em 2009 e 2010, sendo igual ou superior a $94 \mathrm{~cm}$ para homens e $80 \mathrm{~cm}$ para mulheres.

Após análise dos dados coletados, foram definidos os temas que seriam abordados na intervenção nutricional educativa, juntamente à nutricionista, levando em consideração a necessidade do local. Sendo assim, os dois temas elencados foram: "alimentação saudável e a importância dos micronutrientes" e "hidratação e ingestão de líquidos".

A primeira atividade da educação nutricional foi proposta a fim de saber o nível de conhecimento dos idosos sobre alimentação saudável, e, para tanto, disponibilizou-se um prato plástico e recortes variados de modelos alimentares inseridos na realidade alimentar desta comunidade aos oito idosos que se mostraram interessados na atividade, sendo seis do sexo feminino e dois do masculino, divididos em pequenos grupos ou individualmente. Solicitou-se que colocassem, no prato, os alimentos tidos por eles como saudáveis, deixando-os livres para a escolha de quais e quantos modelos seriam depositados no prato. Entre os recortes, havia água, arroz, batata, farinha de trigo, pão francês, milho, macarrão, vários tipos de frutas e hortaliças, leite, queijo, ovos, carne branca e vermelha, feijão, ervilha, margarina, óleo de soja, açúcar e biscoito recheado. Após esta primeira etapa, com o auxílio de uma pirâmide alimentar adaptada às necessidades destes idosos e elaborada com os mesmos modelos alimentares, baseada na proposta de pirâmide de Philippi et al. (1999), a pesquisadora introduziu conceitos de alimentação saudável e a importância dos micronutrientes. 
A segunda atividade foi destinada à hidratação e ingestão de líquidos com uma breve exposição sobre o tema (funções da água, sua atuação no organismo, em que ela ajuda, sintomas da desidratação e suas consequências) utilizando recurso visual e comunicação oral, e contou com a presença de dezoito idosos. Posteriormente, cada idoso ganhou uma garrafa (plástica, de 510mL) de água e, com auxílio da pesquisadora, pôde personalizá-la utilizando fita adesiva colorida e caneta do tipo marcador permanente. Esta atividade foi pensada com o intuito de incentivar o consumo diário de água.

A penúltima semana foi dedicada à cozinheira e aos responsáveis pela alimentação dos idosos a fim de trabalhar conceitos com vistas a aprimorar o cardápio dentro da realidade do local e tornar o ambiente e a hora da refeição mais agradável aos internos. Para tanto, foi discutido, junto à nutricionista, após análise do cardápio, o que poderia ser melhorado e como isso poderia ser feito. Em seguida, foi realizada uma conversa com as cozinheiras sobre as ideias propostas.

Para finalizar, como forma de avaliação da ação de educação nutricional, foi realizada uma roda de conversa, um diálogo informal, com todos os idosos a fim de saber suas impressões sobre os encontros e as atividades propostas. O relato e observações do processo educativo foram registrados em diário de campo, o que possibilitou a descrição dos resultados do presente estudo.

Este estudo foi submetido ao CONEP, via Plataforma Brasil, foi analisado pelo Comitê de Ética da Faculdade de Medicina da Unicamp e recebeu aprovação (processo n. 62363). Todos os idosos consentiram em participar mediante assinatura do Termo de Consentimento Livre e Esclarecido.

\section{Resultados}

Os 23 idosos institucionalizados foram convidados a participar de todas as atividades do estudo e foram incluídos nas mesmas de acordo com a sua disponibilidade, vontade e aptidão. A faixa etária do grupo variou de 69 a 80 anos de idade, sendo 13 mulheres e 10 homens.

A primeira atividade realizada foi a observação do cardápio ofertado aos idosos, cardápio este que não sofre grandes variações uma vez que os alimentos servidos provém de doações. Na maioria das vezes, o mesmo é composto por café com leite ou achocolatado, biscoito doce ou salgado, ou pão francês com margarina no desjejum e lanche da tarde; suco de laranja natural no lanche da manhã; arroz, feijão, um prato proteico, uma guarnição e salada, geralmente de folhas colhidas da própria horta do local, no almoço; uma sopa de legumes variados ou macarrão ao molho no jantar; e chá ou leite na ceia. 
Neste ponto, podemos perceber que a vulnerabilidade social reflete na insegurança alimentar uma vez que há pouca variação na dieta dos idosos, diretamente associada à falta de responsabilidade social já que a maior parte dos alimentos é proveniente de doação.

O valor energético total (VET) encontrado foi aproximadamente de 1500 kcal a $1600 \mathrm{kcal}$ e apresentou distribuição adequada para macronutrientes (carboidratos, proteínas e lipídios), limítrofe ou inferior a recomendada para micronutrientes (vitaminas e sais minerais) e fibras, e aumentada para gordura saturada, com cálculo feito pelo software cuja base é a Recommended Dietary Intakes - RDI (NATIONAL HEALTH AND MEDICAL RESEARCH COUNCIL, 1991). As vitaminas mais discrepantes são: vitamina $D$, vitamina $E$, vitamina B6 e folato; e, entre os minerais, estão magnésio, cobre, selênio, manganês e potássio; todos estes com menos da metade do valor de referência recomendado.

As refeições são realizadas em refeitório arejado, claro e agradável, com exceção da colação, onde eles não necessariamente se reúnem à mesa para tomar o suco. $\mathrm{O}$ ato de alimentar-se é cercado de afeto, cuidado e interação entre eles. A alimentação é feita com calma, sendo que os que costumam comer mais rápido são os idosos que não possuem dentição completa. Dificilmente é pedido que coloque mais alimento no prato, sendo o ofertado suficiente. Observou-se que algumas vezes a televisão do local estava ligada, o que casualmente desviava o foco da refeição para o aparelho.

Dos dez idosos que aceitaram participar e estavam aptos para a avaliação antropométrica, dois homens apresentavam-se em eutrofia e dois com sobrepeso, e, desta amostra, todos apresentavam risco de complicações metabólicas aumentado, segundo seus perímetros abdominais, como mostra a Tabela 1. Por outro lado, das mulheres, três apresentavam baixo peso e outras três sobrepeso. Dentre as senhoras com sobrepeso, seus perímetros abdominais apontavam risco aumentado para complicações metabólicas (Tabela 2).

Tabela 1 - Classificação do estado nutricional e risco de complicação metabólica para o sexo masculino.

\begin{tabular}{c|c|c|c|c}
\hline Indivíduo & $\begin{array}{c}\text { IMC } \\
\left.\mathbf{( k g} / \mathbf{m}^{2}\right)\end{array}$ & $\begin{array}{c}\text { Classificação } \\
\text { segundo IMC }\end{array}$ & $\begin{array}{c}\text { Circunferência } \\
\text { abdominal } \mathbf{( c m})\end{array}$ & $\begin{array}{c}\text { Risco de compli- } \\
\text { cação metabólica }\end{array}$ \\
\hline 1 & 34,4 & Sobrepeso & 116,5 & Aumentado \\
\hline 2 & 27,6 & Sobrepeso & 99 & Aumentado \\
\hline 3 & 26,6 & Eutrófico & 100,8 & Aumentado \\
\hline 4 & 22,8 & Eutrófico & 91,5 & Aumentado \\
\hline
\end{tabular}


Tabela 2 - Classificação do estado nutricional e risco de complicação metabólica para o sexo feminino.

\begin{tabular}{|c|c|c|c|c|}
\hline Indivíduo & $\begin{array}{l}\text { IMC } \\
\left(\mathrm{kg} / \mathrm{m}^{2}\right)\end{array}$ & $\begin{array}{l}\text { Classificação } \\
\text { segundo IMC }\end{array}$ & $\begin{array}{l}\text { Circunferência } \\
\text { abdominal }(\mathrm{cm})\end{array}$ & $\begin{array}{l}\text { Risco de compli- } \\
\text { cação metabólica }\end{array}$ \\
\hline 1 & 18,4 & Baixo peso & 66 & Baixo \\
\hline 2 & 19,8 & Baixo peso & 70 & Baixo \\
\hline 3 & 21,3 & Baixo peso & 69 & Baixo \\
\hline 4 & 30,0 & Sobrepeso & 99,6 & Aumentado \\
\hline 5 & 30,2 & Sobrepeso & 98 & Aumentado \\
\hline 6 & 44,3 & Sobrepeso & 120 & Aumentado \\
\hline
\end{tabular}

$\mathrm{Na}$ atividade com a pirâmide alimentar, oito idosos quiseram participar. O primeiro grupo era composto por cinco senhoras que, após analisarem as figuras, decidiram que todos os alimentos eram saudáveis e, portanto, colocaram todos no prato. No trabalho com a pirâmide alimentar, os grupos das frutas e hortaliças foram os que mais chamaram a atenção das senhoras devido à importância dos micronutrientes, e elas faziam questão de salientar que sempre consumiam estes grupos alimentares.

Estas mesmas etapas foram repetidas com um senhor que também colocou todos os modelos no prato e, quando trabalhada a pirâmide, apenas prestava atenção e repetia as informações sem uma ênfase específica no terceiro grupo, composto por uma senhora que fez a atividade e um senhor que preferiu apenas observar, não foram incluídos no prato: ovos, milho, macarrão, biscoito recheado e carnes. Quando questionado o porquê, apenas relatou que o ovo não era saudável, pois continha muito colesterol e que não havia incluído outros alimentos por questão de gosto. Posteriormente, foi trabalhada a pirâmide, com indicação de onde cada alimento deixado de fora do prato se encaixaria e suas propriedades, desmistificando o suposto malefício que o ovo traria à saúde e pontuando seus benefícios se consumido com moderação.

O tema hidratação e ingestão de líquidos era o mais aguardado, pois há grande dificuldade de ingestão de líquido por este grupo. Sendo assim, dezoito idosos se interessaram por participar e apenas um recusou-se dizendo o seguinte: "Eu não bebo água, desse mal eu não sofro". Foi utilizado o recurso visual para a explicação do tema individualmente ou a grupos reduzidos de até cinco pessoas. Todos prestaram atenção e, logo após o término da fala, ganharam a garrafa de água e fizeram questão de bebê-la em seguida. O que mais atraiu os idosos nessa atividade foi a customização das garrafas. 
Na última atividade, direcionada à cozinheira, trabalhou-se formas de incorporar hortaliças em preparações como feijão e arroz, não incentivar o consumo de café em especial após as refeições principais, bater o suco de laranja com alguma outra fruta ou hortaliça, desligar a televisão no momento da refeição, incentivar o consumo de água e oferecer os alimentos sempre bem cozidos e em pequenos pedaços. Algumas mudanças foram detectadas, pois os alimentos estão sendo cortados em menores pedaços, o café é ofertado apenas aos idosos que o solicitam e há um constante incentivo ao consumo de líquidos.

Na semana reservada para as despedidas e avaliação somativa, perguntou-se aos idosos se eles haviam gostado das atividades. Todos afirmaram, de forma positiva, que os encontros foram válidos e que estão colocando em prática o que aprenderam.

\section{Discussão}

O presente trabalho buscou, por meio de uma intervenção nutricional educativa, promover hábitos alimentares saudáveis entre idosos. Inicialmente, foi realizada uma análise do cardápio. Observou-se que o valor energético total (VET) do que é ofertado durante um dia foi de 1500 a $1600 \mathrm{kcal}$. Valores semelhantes foram encontrados por Menezes e Marucci (2006), em estudo que utilizou de uma metodologia mais precisa, a pesagem direta dos alimentos oferecidos. Neste estudo, o VET médio foi de 1571,4 kcal para as mulheres, $1682,9 \mathrm{kcal}$ para os homens e $1604,5 \mathrm{kcal}$ para ambos os sexos.

Em estudo realizado por Menezes, Souza e Marucci (2009), foi aplicado o recordatório do tipo 24 horas, e verificou-se VET de 1475,8 kcal para homens e 1236,4 kcal para mulheres, sendo a média dos dois de 1316,3 kcal. Possivelmente, a dieta praticada pelos idosos, em termos de VET, tanto no presente estudo como nos demais, está abaixo da necessidade energética estimada (NEE) para as idades. Deve se considerar que a ingestão alimentar sofre influência da variabilidade do cardápio, bem como os resultados dependem da padronização da metodologia utilizada, do preparo do pesquisador/entrevistador, e da memória do entrevistado, sendo que, para este último tópico, pode ocorrer um subrelato, uma vez que depende apenas da lembrança do entrevistado e, em se tratando de idosos, podem ocorrer mais falhas quanto a este item.

Com o passar dos anos, há a diminuição da taxa de metabolismo basal (TMB), bem como da necessidade energética cerca de $3 \%$ por década, segundo Mahan e Escott-Stump (2010). Logo, as quantidades ofertadas e ingeridas e o valor nutricional adequado das refeições apresentam relação direta no que diz respeito à alimentação do idoso (CARDOSO, 2004). Vale salientar que há poucos 
estudos sobre educação nutricional voltados aos idosos na literatura, contudo, deveria ser dada maior atenção ao tema e principalmente a esta população uma vez que a expectativa de vida do brasileiro cresce a cada ano, segundo o IBGE (2011).

Além do aporte energético inadequado, outra questão foi a da baixa ingestão de micronutrientes. Dentre estes, os mais discrepantes foram as vitaminas D, E, B6 e folato; e os seguintes minerais: magnésio, cobre, selênio, manganês e potássio. Uma dieta com teor adequado de vitaminas e minerais é essencial para manter diversas funções metabólicas do organismo, tendo o potencial de evitar certas carências causadas pelos micronutrientes (VELÁSQUEZ-MELÉNDEZ et al., 1997).

A deficiência de vitamina D é uma grande preocupação neste grupo. A ingestão inadequada e a pouca exposição ao sol, combinada com a baixa absorção decorrente da idade avançada, podem levar à osteoporose e ao aumento do risco de fraturas (MORIGUTI et al., 2009). A maior parte das deficiências causadas pelos micronutrientes citados pode ser evitada com o aumento do consumo de hortaliças verdes folhosas, leguminosas e carnes bovina e de aves, cuja observação do presente estudo mostrou que estes grupos alimentares aparecem com pouca frequência no cardápio ou muitas das vezes são rejeitados pelos idosos. Para tanto, como medida de prevenção, foi explicado aos idosos à importância de consumi-los, bem como foram dadas dicas à cozinheira do local de como inseri-los na alimentação.

Quanto à antropometria, os resultados são os mais variados dependendo do local pesquisado, ressaltando novamente que o grupo dos idosos é heterogêneo e, portanto, cada indivíduo deve ser visto como tal. No presente estudo, dos dez idosos, cinco foram classificados, segundo o IMC, com sobrepeso. Este resultado também foi encontrado em trabalho realizado por Bueno et al. (2008) com idosos pertencentes a um programa assistencial. Dos idosos com sobrepeso, a maioria era do sexo feminino, bem como o ocorrido na Pesquisa Nacional sobre Saúde e Nutrição (PNSN) (TAVARES; ANJOS, 1999), na qual, para o sexo masculino, temos 61,8\% em eutrofia, seguido de 30,4\% de sobrepeso, e no presente estudo, os eutróficos foram encontrados apenas no grupo dos homens, sendo 50\% eutróficos e 50\% com sobrepeso. Para a população de idosos que se encontram em instituições, os resultados apontavam para uma maior propensão à eutrofia (MENEZES; MARUCCI, 2005; HARDT et al., 2006; GALESI et al., 2008). O mesmo não pode ser corroborado no presente estudo devido à pequena amostra.

Para a educação nutricional foi escolhida a pirâmide alimentar como principal ferramenta por se tratar de uma ferramenta dinâmica e adaptável às especificidades dos grupos populacionais (PHILIPPI et al., 1999). Outros 
estudos destinados à população idosa também utilizaram-se desta ferramenta por ser de fácil explicação e entendimento para este grupo (KIM; KIM; LEE, 2012; CRISCUOLO, 2009; LEE et al., 2004).

Um estudo mais amplo realizado na Argentina, onde foram ministradas 900 oficinas de educação nutricional para idosos, teve objetivo semelhante ao do presente estudo, no que diz respeito à qualidade de vida e incentivo à integração entre os idosos. Quanto à metodologia, também foram utilizados materiais expositivos, dinâmicas e conversas informais para fixar os conteúdos programados especificamente para cada grupo de reunião. O estudo de Guarino et al. (1999) fez parte de um programa de assistência alimentar voltado aos idosos mais carentes, junto ao Ministério do Desenvolvimento Social. Portanto, o trabalho foi bem mais abrangente, embora com o mesmo princípio e resultado semelhante, qual seja o interesse dos idosos em participar e colocar em prática os conhecimentos recebidos, mostrando-se a educação nutricional, mais uma vez, como sendo eficaz para este público.

Outro exemplo de intervenção educativa bem sucedido foi o trabalho realizado por Cervato et al. (2005) com idosos mais independentes e com idade menos avançada que os idosos do recanto, que participaram de um projeto chamado "Universidades Abertas para a Terceira Idade". Utilizou-se como metodologia questionários auto-aplicados e registros alimentares de três dias, e, após analisados estes dados, foi feita a educação nutricional por meio de uma atividade que consistia em duas partes: uma orientação geral (aulas sobre nutrição) e uma específica (orientação individual). Quanto ao resultado, observou-se diferenças estatisticamente significativas em relação aos conceitos sobre nutrição, refletidos em modificações na dieta e aumento da ingestão de água. A educação nutricional, em todos os estudos, mostrou-se como instrumento promotor da independência dos idosos, uma vez que por meio da problematização e da discussão de conceitos, bem como atividades práticas, eles se empoderam para fazer melhores escolhas alimentares, com vistas à promoção da própria saúde e de sua qualidade de vida.

\section{Conclusão}

Os idosos do recanto Nossa Senhora do Rosário apresentaram dieta com um valor energético inferior ao estimado para a idade e, mesmo assim, muitos se encontram em estado de sobrepeso, o que pode ser causado pela baixa atividade física combinada com o uso de medicamentos variados. Em relação à educação nutricional, esta se mostrou uma experiência válida, sendo possível perceber mudanças sutis, embora importantes, logo nas semanas seguintes às atividades aplicadas. 
NUTRITION EDUCATION FOR INSTITUTIONALIZED ELDERLY PEOPLE AT THE REST HOME NOSSA SENHORA DO ROSÁRIO IN LIMEIRA, SP

\section{abstract}

Health studies suggest it is possible to achieve through health promotion and disease prevention a healthy aging, preserving the functional capacity of the elderly as long as possible. The aim of the present study is to promote, through activities of Nutrition Education, healthy eating habits in a group of institutionalized elderly in the city of Limeira, São Paulo. It was an intervention study consisting of a sample of 23 elderly, 13 women and 10 men, with age ranging from 69 to 80 years, residents at Nossa Senhora do Rosário. Weekly visits were established during seven weeks, totaling seven meetings, including two for observation of the menu and meals, one for anthropometry, two for nutritional education, one for activity with the responsible for preparing meals and one for final consideration and socializing. In the first two weeks, we observed the usual menu of the rest home from the preparation until the time the meal was served. The total energy was around $1500 \mathrm{kcal}$ to $1600 \mathrm{kcal}$, and proper distribution of macronutrients, limitrophe or below for recommended micronutrients and fiber, and increased to saturated fat. Only ten elderly were able and agreed to participate in the anthropometric evaluation, with five of them being overweight. The activity with the food pyramid involved eight elderly. The hydration and liquid intake theme was the most awaited by the group due to the difficulty related to the them. Eighteen elderly were interested in participating in this activity. Nutritional education proved to be a worthwhile experience, being possible to notice subtle, although important changes, right after the weeks following the applied activities.

keywords

Food and Nutritional Education. Elderly Nutrition. Health of the Elderly. Health of Institutionalized Elderly.

\section{referências}

\footnotetext{
ALENCAR, Maria do Socorro S.; BARROS JúNIOR, Francisco de O.; CARVALHO, Cecília Maria R. G. Os aportes sócio-políticos da educação nutricional na perspectiva de um enveIhecimento saudável. Revista de Nutrição, Campinas, v. 21, n. 4, p. 369-381, jul./ago. 2008.

ASSIS, Mônica de (Org.). et al. Promoção da saúde e envelhecimento: orientações para o desenvolvimento de ações educativas com idosos. Rio de Janeiro: CRDE UnATI UERJ, 2002. Disponível em: <http://www.crde-unati.uerj.br/publicacoes/pdf/ promocao_da_saude.pdf>. Acesso em: 4 maio 2012.
} 
BRASIL. Ministério da Saúde. SISVAN - Notas técnicas. Disponível em <http://tabnet. datasus.gov.br/cgi-win/SISVAN/CNV/notas_sisvan.html>. Acesso em: 23 set. 2012.

Ministério da Saúde. Vigilância de doenças crônicas não transmissíveis. Disponível em: <http://portal.saude.gov.br/portal/saude/profissional/visualizar_texto. cfm?idtxt=31877>. Acesso em: 1 maio 2012.

BUENO, Júlia M. et al. Avaliação nutricional e prevalência de doenças crônicas não transmissíveis em idosos pertencentes a um grupo assistencial. Ciência e Saúde Coletiva, Rio de Janeiro, v. 13, n. 4, p. 1237-1246, jul./ago. 2008.

CARDOSO, Marco Rogério V. Alimentação e estado nutricional de idosos residentes em instituições asilares de dois municípios do sul de Minas Gerais. 2004. 113 f. Tese (Mestrado em Ciências dos Alimentos) - Universidade Federal de Lavras, Lavras. 2004.

CARNEIRO, Rachel S. et al. Qualidade de vida, apoio social e depressão em idosos: relação com habilidades sociais. Psicologia: Reflexo e Crítica, Porto Alegre, v. 20, n. 2, p. 229-237, 2007.

CERVATO, Ana Maria et al. Educação nutricional para adultos e idosos: uma experiência positiva em Universidade Aberta para a Terceira Idade. Revista de Nutrição, Campinas, v. 18, n. 1, p. 41-52, jan./fev. 2005.

COLÉGIO SÃO BENEDITO. A Paróquia de São Benedito. Disponível em: <http://saobeneditolimeira.com.br/paroquia-sao-benedito>. Acesso em: 3 maio 2014.

COSTA, Letícia C.; THULER, Luiz Claudio S. Fatores associados ao risco para doenças não transmissíveis em adultos: estudo transversal de base populacional. Revista Brasileira de Estudos de População, Rio de Janeiro, v. 29, n. 1, p. 133-145, 2012.

CRISCUOLO, Carolina. Contribuições da educação nutricional junto a um grupo de idosos. 2009. 199 f. Tese (Mestrado em Alimentos e Nutrição) - Faculdade de Ciências Farmacêuticas, Universidade Estadual Paulista "Júlio de Mesquita Filho", Araraquara. 2009.

FERREIRA, Lucas L. et al. Perfil sociodemográfico e funcional de idosos institucionalizados. Estudos Interdisciplinares sobre o Envelhecimento, Porto Alegre, v. 17, n. 2, p. 373-386, 2012.

FREIRE JÚNIOR, Renato C.; TAVARES, Maria de Fátima L. A saúde sob o olhar do idoso institucionalizado: conhecendo e valorizando sua opinião. Interface - Comunicação, Saúde, Educação, Botucatu, v. 9, n. 16, p. 147-158, set./fev. 2005.

GALESI, Lilian F. et al. Perfil alimentar e nutricional de idosos residentes em moradias individuais numa instituição de longa permanência no leste do estado de São Paulo. Alimentos e Nutrição, Araraquara, v. 19, n. 3, p. 283-290, jul./set. 2008.

GUARINO, Maria de los Angeles et al. Talleres de Educación Alimentaria Nutricional (EAN) con ancianos: una actividad convocante que complementa a um programa de asistencia alimentaria. In: ENCUENTRO NACIONAL SOBRE CALIDAD DE VIDA EM LA TERCERA EDAD, 1, 1999, Buenos Aires. Anais... . Buenos Aires. Disponível em: <http:// www.naya.org.ar/congresos/contenido/3ra_edad/2/18.htm>. Acesso em: 7 nov. 2012.

HARDT, Ana Carolina et al. Um olhar a terceira idade: promovendo nutrição e saúde no asilo Irmão Joaquim. In: ENCONTRO NACIONAL DOS GRUPOS PET, 11., 2006, Florianópolis. Anais... . Florianópolis: Editora UFSC, 2006.

INSTITUTO BRASILEIRO DE GEOGRAFIA E ESTATÍSTICA. Brasil: tábua completa de mortalidade - 2010. Rio de Janeiro, 2011.

KIM, Bok Hee; KIM, Mi-Ju; LEE, Yoonna. The effect of a nutritional education program on the nutritional status of elderly patients in a long-term care hospital in Jeollanamdo province: health behavior; dietary behavior; nutritional risk level and nutrient intake. Nutrition Research and Practice, Seoul, v. 6, n. 1, p. 35-44, Feb. 2012. 
LEBRÃO, Maria Lúcia. O envelhecimento no Brasil: aspectos da transição demográfica e epidemiológica. Saúde Coletiva, São Paulo, v. 4, n. 17, p. 134-140, 2007.

LEE, Jung $S$. et al. Edentulism and nutritional status in a biracial sample of weel-functioning, community-dwelling elderly: the Health, Aging, and Body Composition Study. American Journal of Clinical Nutrition, New York, n. 79, p. 295-302, 2004.

MAHAN, L. Kathleen; ESCOTT-STUMP, Sylvia. Krause, Alimentos, Nutrição e Dietoterapia. 12. ed. São Paulo: Elsevier, 2010.

MENEZES, Tarciana N.; MARUCCI, Maria de Fátima N. Antropometria de idosos residentes em instituições geriátricas, Fortaleza, CE. Revista Saúde Pública, São Paulo, v. 39, n. 2, p. 169-175, 2005.

MENEZES, Tarciana N.; MARUCCI, Maria de Fátima N. Oferta e consumo alimentar de idosos residentes em instituições geriátricas: diferença no valor energético total. Fortaleza/Ceará. Nutrire: Revista da Sociedade Brasileira de Alimentação e Nutrição, São Paulo, v. 31, n. 2, p. 1-11, ago. 2006.

MENEZES, Tarciana N.; SOUZA, José M. P.; MARUCCI, Maria de Fátima N. Necessidade energética estimada, valor energético e adequação de macronutrientes da alimentação dos idosos de Fortaleza/CE. Nutrire: Revista da Sociedade Brasileira de Alimentação e Nutrição, São Paulo, v. 34, n. 3, p. 17-30, dez. 2009.

MORIGUTI, Julio César et al. Nutrição no idoso. In: LOPES, Antônio Carlos (Org.). Tratado de Clínica Médica. 2. ed. São Paulo: Roca, v. 3, p. 4273-4281, 2009.

NATIONAL HEALTH AND MEDICAL RESEARCH COUNCIL. Recommended Dietary Intakes for use in Australia. Australian Government Publishing Service, Canberra, 1991.

PAULO, Thais R. S. de et al. O exercício físico funcional para idosos institucionalizados: um novo olhar para as atividades da vida diária. Estudos Interdisciplinares sobre o Envelhecimento, Porto Alegre, v. 17, n. 2, p. 413-427, 2012.

PHILIPPI, Sônia T. et al. Pirâmide alimentar adaptada: guia para escolha dos alimentos. Revista de Nutrição, Campinas, v. 12, n. 1, p. 65-80, jan./abr. 1999.

SILVA, Marilza Francisca B. da. Fatores Determinantes do Asilamento de Idosos. 2007. 37 f. Tese (Bacharel em Serviço Social) - Escola Superior de Ciências da Santa Casa de Misericórdia, Vitória. 2007.

TAVARES, Elda L.; ANJOS, Luiz Antonio dos. Perfil antropométrico da população idosa brasileira. Resultados da Pesquisa Nacional sobre Saúde e Nutrição. Cadernos de Saúde Pública, Rio de Janeiro, v. 15, n. 4, p. 759-768, out./dez. 1999.

VELÁSQUEZ-MELÉNDEZ, Gustavo et al. Consumo alimentar de vitaminas e minerais em adultos residentes em área metropolitana de São Paulo, Brasil. Revista de Saúde Pública, São Paulo, v. 31, n. 2, p. 157-162, abr. 1997.

VITORELI, Eliane; PESSINI, Salete; SILVA, Maria Júlia P. da. A auto-estima de idosos e as doenças crônico-degenerativas. Revista Brasileira de Ciências do Envelhecimento Humano, Passo Fundo, v. 2, n. 1, p. 102-114, jan./jun. 2005.

WATANABE, Helena A. W. Instituições de Longa Permanência para Idosos. In: WATANABE, Helena A. W. et al. (Org). Rede de Atenção à Pessoa Idosa. São Paulo: Secretaria Estadual de Assistência e Desenvolvimento Social: Fundação Padre Anchieta, 2009.

WORLD HEALTH ORGANIZATION. Definition of an older or elderly person. 2010. Disponível em: <http://who.int/healthinfo/survey/ageingdefnolder/en>. Envelhecimento ativo: uma política de saúde. Tradução de Suzana Gontijo. Brasília: Organização Pan-Anamericana da Saúde, 2005. 